\title{
Sistema para Transição de Governos no Brasil
}

\author{
Débora Lima $^{1}$, Ricardo Silva ${ }^{1}$, Ana Garcia ${ }^{1}$, Sérgio Rodrigues ${ }^{1}$, Miriam Chaves ${ }^{2}$, \\ Rodrigo Santos ${ }^{1}$, Jano Souza ${ }^{1}$ \\ ${ }^{1}$ PESC/COPPE - Universidade Federal do Rio de Janeiro (UFRJ) \\ Rio de Janeiro, RJ - Brasil \\ ${ }^{2}$ MPOG - Esplanada dos Ministérios - Bloco K - $4^{\mathrm{o}}$ andar \\ Brasília, DF - Brasil \\ $\{$ dlima, rick, sergio, rps, jano\}@cos.ufrj.br, anacog@pobox.com,
miriam.chaves@planejamento.gov.br
}

\begin{abstract}
Changes in teams always represent a challenge for organizations and societies. In the governmental domain, this is also critical. In the Brazilian Govern case, the teams have a short time (120 days) to accomplish the political transition and they usually have distinct points of view. This way, information systems have become important pieces to aid the knowledge management applied to different governmental systems as well as their integration. This paper presents a system that works as a central piece for joining information from many levels of Brazilian Govern's federal sphere. This system extracts and organizes governmental transition reports in order to contribute to programs and initiatives continuity.
\end{abstract}

Resumo. Realizar mudanças entre equipes representa sempre um desafio. No âmbito governamental, isso não poderia ser diferente. As equipes do Governo do Brasil, às vezes com pontos de vista extremamente distintos, têm um curto período de tempo (120 dias) para conseguirem realizar a transição política. Assim sendo, sistemas de informação que auxiliem na gestão de conhecimento dos diversos sistemas do Governo se tornam cada vez mais necessários. Este artigo apresenta um sistema que provê um ponto único para reunião de informações de diversos niveis da esfera federal do Governo do Brasil ao extrair e organizar relatórios de transição governamental. Espera-se, com isso, contribuir para a continuidade programas e iniciativas.

\section{Introdução}

A pesquisa em governo eletrônico vem despertando interesse da comunidade de pesquisadores em Computação há aproximadamente uma década, devido aos impactos trazidos pelas tecnologias Web, embora ainda esteja em um estágio inicial (WCGE, 2012). Com o crescente número de publicações sobre o assunto no cenário nacional, internacionalmente, a maior parte das publicações se concentra em poucos países, com destaque para áreas como Ciências Sociais, Ciência da Informação e Ciência da Computação. Isso embasa o fato de que a pesquisa para apoiar processos gerenciais nos governos está sendo tratada pelas diferentes áreas de conhecimento. Uma das mais recentes utilizações de sistemas computacionais para este fim consiste no suporte ao 
planejamento, controle e acompanhamento de processos que compõem as instituições governamentais (Nascimento et al., 2009; Preti et al., 2010; Silva \& Ralha, 2010).

Nesse contexto, a aplicação de sistemas de informação em governo eletrônico é denominada de e-gov, ou seja, a utilização de tecnologias da informação e da comunicação (TICs) baseadas na Web visando melhorar a eficiência e a efetividade na entrega de serviços no setor público (Jeong, 2007). Mais especificamente no caso brasileiro, o núcleo central de gestão administrativa do Governo Federal compreende um conjunto de sistemas de informação: Sistemas de Gestão Administrativa ou Sistemas Estruturadores de Governo (Governo do Brasil, 2012). O foco de cada um está sobre um processo de gestão específico, e.g., elaboração/acompanhamento do orçamento, administração de recursos humanos, alocação de cargos, compras governamentais, acompanhamento dos programas de governo, administração financeira e contábil etc. Por outro lado, sistemas corporativos apoiam as áreas da saúde, planejamento e receita e vêm recebendo dados dos Sistemas Estruturadores para apoiar as ações de Governo de forma mais integrada, com melhor qualidade e com base em uma arquitetura referencial (Franzosi et al., 2009).

Uma vez que os sistemas monolíticos do Governo Federal passam a ser integrados com o uso de arquiteturas interoperáveis baseadas em serviços, a transição governamental pode sofrer algum tipo de impacto. A cada eleição e, consequentemente, troca de equipes de governo, torna-se necessária a criação do chamado "Livro da Transição". Este livro corresponde a um resumo do status atual de um determinado órgão do Governo Federal. Ele contém informações orçamentárias, de recursos humanos e de diversas outras visões administrativas - um resumo das informações nele contidas é apresentada posteriormente neste artigo. Para a elaboração deste livro, um esforço considerável se faz necessário, em se tratando da forte heterogeneidade dos sistemas que servem como entrada de dados, bem como da diagramação necessária para a padronização do livro (Silva \& Ralha, 2010).

A fim de favorecer o processo de construção do Livro da Transição, este artigo apresenta o resultado de um projeto de pesquisa e desenvolvimento de um sistema de informação denominado Sistema de Transição governamental. Este sistema nasceu com o objetivo de integrar as diversas fontes de dados necessárias, complementar os dados oriundos dos responsáveis por cada Órgão/Secretaria do Governo Federal e, por fim, gerar o Livro da Transição de forma automatizada, dentro do padrão estabelecido. Além desta seção de introdução, o artigo está estruturado da seguinte forma: a Seção 2 discorre sobre governo eletrônico e integração de sistemas; a Seção 3 discute o Sistema de Transição a partir de seus requisitos, arquitetura e interfaces de comunicação; a Seção 4 apresenta telas de exemplos de implantação, uso e manutenção do Sistema de Transição; e a Seção 5 conclui o artigo a aponta trabalhos futuros.

\section{Revisão da Literatura}

A maior parte dos sistemas do Governo Federal, principalmente os estruturadores, foi desenvolvida até os anos 80 com base em mainframes, em antigas linguagens de programação e em padrões proprietários (Franzosi et al., 2009). Do ponto de vista de processo de software, os sistemas estruturadores da Administração Pública levam de quatro a cinco anos para serem implantados, cerca de dez anos para atingir a sua 
maturidade e de 20 a 25 anos para serem substituídos por uma nova versão, o que gera um impacto real sobre a efetividade de sua integração (Santanna, 2007). Isso motivou a proposição e implantação da Arquitetura de Padrões de Interoperabilidade de Governo (e-PING) e da Arquitetura Referencial de Interoperabilidade dos Sistemas Informatizados de Governo (AR) (Franzosi et al., 2009).

Nesse sentido, a Estratégia Geral de Tecnologia da Informação do Governo Federal visa estimular a reutilização de componentes e padronizar a sua estrutura de desenvolvimento de software por meio do uso rotineiro de soluções existentes (MPOG, 2012), sobretudo considerando o desenvolvimento de sistemas distribuídos sob as visões estratégica e técnica (Sizo et al., 2009). Adicionalmente, destaca-se a ampliação do uso de software livre pelo Governo Federal pela necessidade de aumentar a segurança ao utilizar software nacional e de romper com a dependência tecnológica e custos desnecessários com licenças, além de representar um investimento da inteligência nacional e às particularidades do caso brasileiro (Tiboni et al., 2009). Isso contribui diretamente para uma experiência inovadora na Administração Pública ao estimular o desenvolvimento de um ecossistema digital, i.e., um ambiente povoado por "espécies digitais" (i.e., componentes, aplicações, serviços etc.) manipuladas por diversos stakeholders reunidos sobre uma plataforma central de e-gov (Santos \& Werner, 2011; Stefanuto et al., 2011). Ou seja, o Governo Federal passa a estabelecer parcerias com centros de pesquisa brasileiros visando o desenvolvimento de software público de acordo com as suas necessidades e peculiaridades.

No âmbito regional e/ou específico de uma área de governo, existem algumas iniciativas para estruturação e/ou integração de sistemas a fim de viabilizar a criação e manutenção de ecossistemas digitais centrados em uma plataforma comum. Isso favorece a interoperabilidade entre os serviços dos diversos sistemas bem como a troca de informação. Por exemplo, o projeto SIGFAPESPA é relativo à construção de um sistema integrado de gestão para a Fundação de Amparo à Pesquisa (FAP) do Estado do Pará (Diniz et al., 2009). O objetivo do sistema está além das questões técnicas, que envolvem a evolução do produto: visa à consolidação do comitê gestor para permitir a expansão do sistema a fim de tratar a participação das demais FAPs que demonstrarem interesse. Outro exemplo é o sistema para gestão e inteligência fiscal (GIF) implantado no Município de Campo Bom (RS), cuja meta é viabilizar a comunicação entre prestadores de serviços e a administração tributária municipal (Nascimento et al., 2009). Isso também vem acontecendo em outros municípios, o que pode caminhar para um sistema nacional integrado no futuro.

Frente aos cenários apresentados, a extração de informação e o processamento de consultas para geração de relatórios a partir de múltiplos sistemas se tornam desafios identificados em uma necessidade real (Santos et al., 2012; TI MAIOR, 2012). Para que as metas governamentais sejam atingidas, como o "Livro da Transição" mencionado na Seção 1 (alvo deste trabalho), algumas questões merecem atenção por parte dos fornecedores de soluções para e-gov no Brasil nos próximos anos: (i) busca semântica e recuperação de informação para diferentes sistemas que compõem as plataformas de ecossistemas digitais em e-gov (Pita \& Paixão, 2010); (ii) apoio colaborativo de várias mídias para ampliar e facilitar a interação (Preti et al., 2010); e (iii) computação nas nuvens aplicada ao domínio do e-gov com foco em serviços e aplicações (Martins, 
2010). A principal motivação está no fato de que, para o Governo Federal, muitas vezes é custoso utilizar soluções não personalizadas (adquiridas externamente).

\section{Sistema de Transição}

Considerando as necessidades descritas anteriormente, a COPPE/UFRJ desenvolveu, em parceria com o Ministério do Planejamento, Orçamento e Gestão (MPOG), o Sistema de Transição. O objetivo deste sistema é centralizar e padronizar a integração entre os diversos dados necessários para a elaboração do Livro da Transição, voltado para as particularidades do caso nacional. Nesta seção, descreve-se como o sistema foi implementado e suas características técnicas. Ressalta-se que o sistema não foi desenvolvido para substituir os processos já existentes, mas apoiá-los, dado que as modificações desses processos costumam esbarrar em questões legais e burocráticas. Além disso, cada ministério é regido por um conjunto de processos, de modo que não existe necessariamente um padrão nos Sistemas Estruturadores/processos adotados.

\subsection{Requisitos de Informação}

O sistema disponibiliza informações estruturais, de orçamento, de síntese organizacional, concursos, colegiados coordenados pelos órgãos, síntese dos resultados alcançados, Plano Plurianual (PPA), projetos suspensos, contratos, obrigações legais, obrigações institucionais, glossário, visão geral da Administração Pública Federal (APF) e contas públicas. A seguir, apresenta-se uma explicação sintética de cada área:

- Estrutura: estrutura hierárquica do órgão, bem como a sua competência, base legal, natureza jurídica e organograma;

- Orçamento: informações sobre o orçamento do órgão ou entidade, separado por grupo de gasto e unidade orçamentária, e a visão geral da execução orçamentária do órgão ou entidade para o último ano do mandato (e.g., 2010);

- Recursos Humanos - Síntese Organizacional: quantitativo do quadro de pessoal do órgão ou entidade, de acordo com o cargo e situações de vínculo, incluindo ainda informações de cargos comissionados e funções gratificadas;

- Recursos Humanos - Concursos: informações sobre os concursos autorizados para o órgão ou entidade, relativas ao provimento de vagas, prazo de validade e situação do concurso, permitindo ainda que o órgão ou entidade informe os concursos solicitados, mas ainda não autorizados;

- Colegiados: informações sobre Comitês, Comissões, Conselhos e Grupos de Trabalho coordenados pelo o órgão ou entidade;

- Ação Governamental - Síntese: informações sobre as prioridades estabelecidas para o primeiro ano do novo mandato, os principais resultados do mandato anterior e os principais avanços dos marcos institucionais e regulatórios no mandato anterior para um órgão ou entidade;

- Ação Governamental - Programa: lista dos Programas do PPA segundo o Sistema de Informações Gerenciais e de Planejamento, que tenha relevância para o órgão ou entidade; 
- Projetos Suspensos: informações sobre as iniciativas que foram suspensas por determinação de órgãos de controle, do Poder Judiciário ou ainda do próprio órgão ou entidade;

- Agenda 120 Dias - Contratos: informações sobre os contratos de serviços gerais e de apoio às atividades finalísticas de relevância para o órgão ou entidade, cuja vigência se encerre nos primeiros 120 dias do primeiro ano do novo mandato, ou cujo processo de contratação futura dependa de etapas a serem implementadas nesse período;

- Agenda 120 Dias - Obrigações Legais: informações sobre as obrigações que derivam de algum ato normativo, incluindo as normas constitucionais e os acordos internacionais;

- Agenda 120 Dias - Obrigações Institucionais: informações sobre os compromissos assumidos pelo órgão e seus dirigentes para com terceiros, que não decorram de obrigação legal ou contratual e cujo descumprimento não acarrete em ilegalidade ou quebra de contrato;

- Glossário: lista de siglas e/ou termos técnicos, acompanhados de suas respectivas definições, comumente utilizados pelo órgão ou entidade;

- Visão Geral Poder Executivo - APF: arquivos da estrutura organizacional da APF (visão geral da estrutura, visão das entidades vinculadas e lista dos Sistemas Estruturadores com respectivos órgãos responsáveis e atos legais) e arquivos com roteiros detalhados para nomeação/exoneração, requisição, deslocamento, diárias e passagens, e orçamento, incluindo um calendário com as datas críticas de orçamento dentro dos 120 dias do novo mandato;

- Contas Públicas: informações a respeito das contas públicas da APF;

- Livro Transição: todas as informações do órgão ou entidade que constam na aplicação, em formato de documento de texto;

- Controle de Usuários: lista de usuários cadastrados no sistema, visando apoiar a inclusão, desativação e atualização dos dados dos usuários.

O Sistema de Transição possui os seguintes perfis de acesso:

- Usuário Setorial, habilitado para atualização das informações do órgão ao qual está associado no sistema;

- Usuário Ministerial: habilitado para atualização das informações do órgão ao qual está vinculado e de toda a sua hierarquia (Administração Direta e Indireta);

- Usuário Global: habilitado para atualização das informações de qualquer órgão;

- Administrador Técnico: autoriza o acesso do usuário, atribuindo-lhe um perfil adequado para a sua atividade.

O sistema também contempla permissões de acesso aos usuários cadastrados: (i) leitura: permitir a visualização das informações do sistema de acordo com seu perfil; e (ii) leitura e escrita: permitir a visualização e alteração das informações de acordo com seu perfil. Por fim, o sistema apresenta os seguintes requisitos de segurança: 
- Protocolo HTTPS: o sistema foi desenvolvido para acesso apenas via HTTPS, almejando assim uma maior segurança;

- Controle de Usuários: o sistema pode ser acessado somente por usuários previamente cadastrados pelo Administrador Técnico. Quando o usuário realizar alguma alteração em dados do sistema, um registro será gerado com base em suas permissões;

- Log de Acesso: todas as transações realizadas geram um log, que contém as seguintes informações: ip_solicitante (IP da máquina que realizou a transação), dsc login usu (login do usuário que realizou a transação), dsc transacao (descrição da transação) e dta_transacao (data em que a transação foi realizada);

- Log de Carga: todas as cargas realizadas na base de dados do sistema geram um registro de carga especificando as fontes e datas da realização, que são disponibilizadas na forma de um informativo de sistema.

\subsection{Arquitetura do Sistema}

O sistema foi desenvolvido com a tecnologia Java Enterprise Edition (JEE), fazendo uso da técnica Model Driven Architecture (MDA) por meio do Framework do Ministério da Defesa e do MPOG, denominado MDArte versão 3.1.1.3.4.12. O MDArte é utilizado no desenvolvimento de aplicações em três camadas, seguindo o padrão arquitetural MVC (Model-View-Controller). As tecnologias utilizadas nesta geração e, consequentemente, no desenvolvimento de alterações incluem, no lado servidor, Linguagem Java, EJB, JSP, JSF, Struts e, no lado cliente, CSS, HTML e JavaScript. A arquitetura de uma aplicação sob o MDArte é sumarizada na Figura 1 e na Tabela 1.

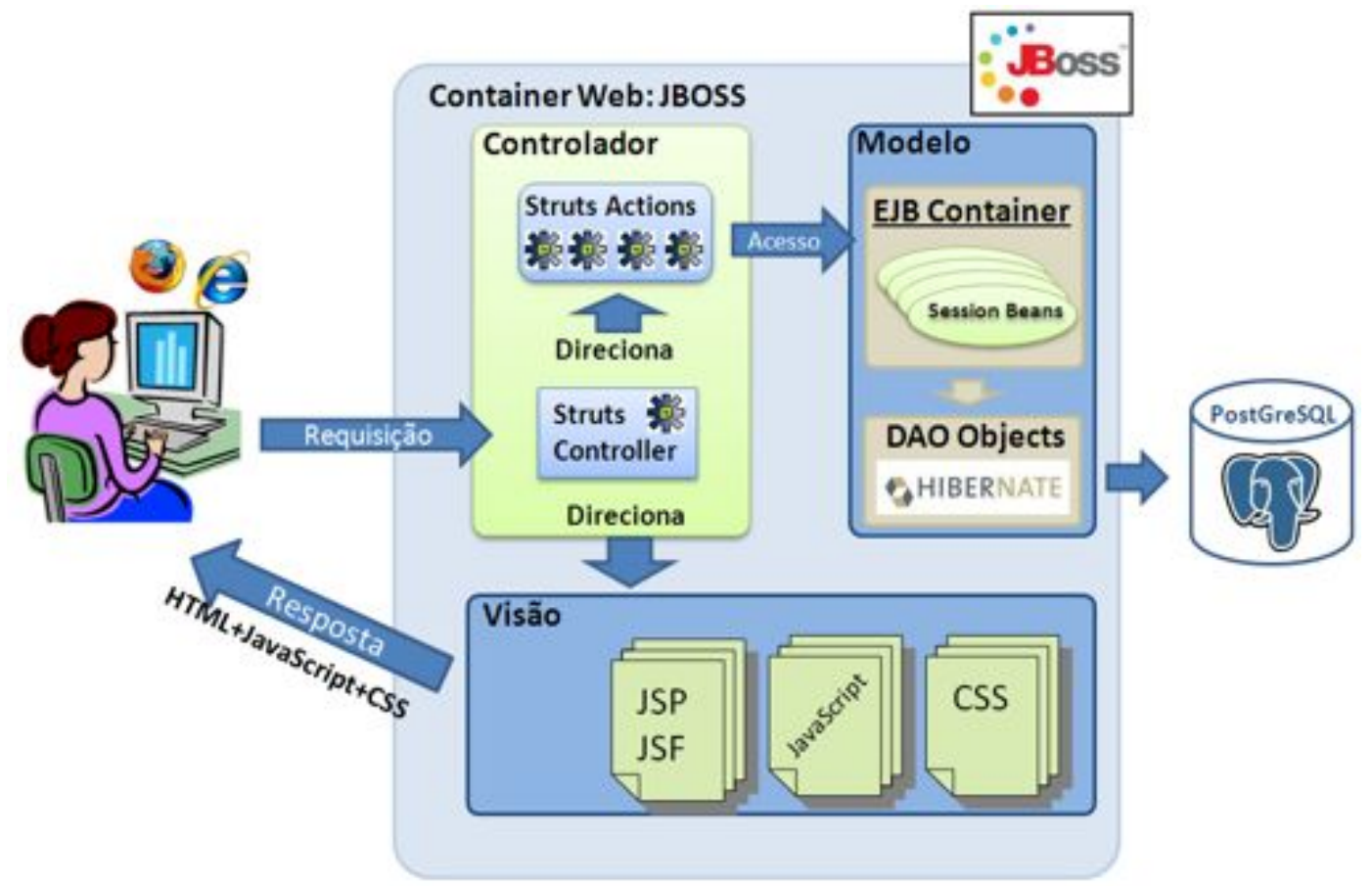

Figura 1. Arquitetura de uma aplicação desenvolvida com o uso do MDArte 
O banco de dados foi construído a partir do Sistema de Gerenciamento de Banco de Dados PostgreSQL. Este foi carregado com dados oriundos de (i) planilhas; (ii) arquivos do Sistema Integrado de Administração de Recursos Humanos (SIAPE), do Sistema Integrado de Administração Financeira (SIAFI) e do Sistema de Informações Gerenciais e de Planejamento (SIGPLAN); e (iii) dados do banco de dados do Sistema de Informações Organizacionais do Governo Federal (SIORG). Este insumo foi obtido por meio de consultas a uma visão disponibilizada no formato MSAccess.

Tabela 1. Resumo dos elementos da aplicação baseada no MDArte

\begin{tabular}{|c|c|}
\hline Operação & Tecnologia \\
\hline Gerador MDA & MDArte \\
\hline Visão (telas da interface) & JSP/JSF/CSS/HTML/JS \\
\hline Controlador & Struts \\
\hline Modelo & $\begin{array}{c}\text { EJB Session Beans/DAO } \\
\text { Objects/Hibernate }\end{array}$ \\
\hline $\begin{array}{c}\text { Banco de dados } \\
\text { Gerencial }\end{array}$ & PostgreSQL \\
\hline Servidor de Aplicação & JBOSS \\
\hline
\end{tabular}

\subsection{Integrações com Outros Sistemas}

De acordo com as necessidades identificadas por meio da Seção 3.1, dados de sistemas diferentes deveriam ser disponibilizados para o Sistema de Transição por meio de serviços. Com este intuito, foi realizado um esforço considerável para manter vínculos de comunicação com os órgãos e setores responsáveis pela manutenção e desenvolvimento desses serviços. A Figura 2 fornece uma visão resumida de quais sistemas são integrados ao Sistema de Transição e do mecanismo criado para esta finalidade. A seguir, a integração de cada um deles ao Sistema de Transição é explicada.

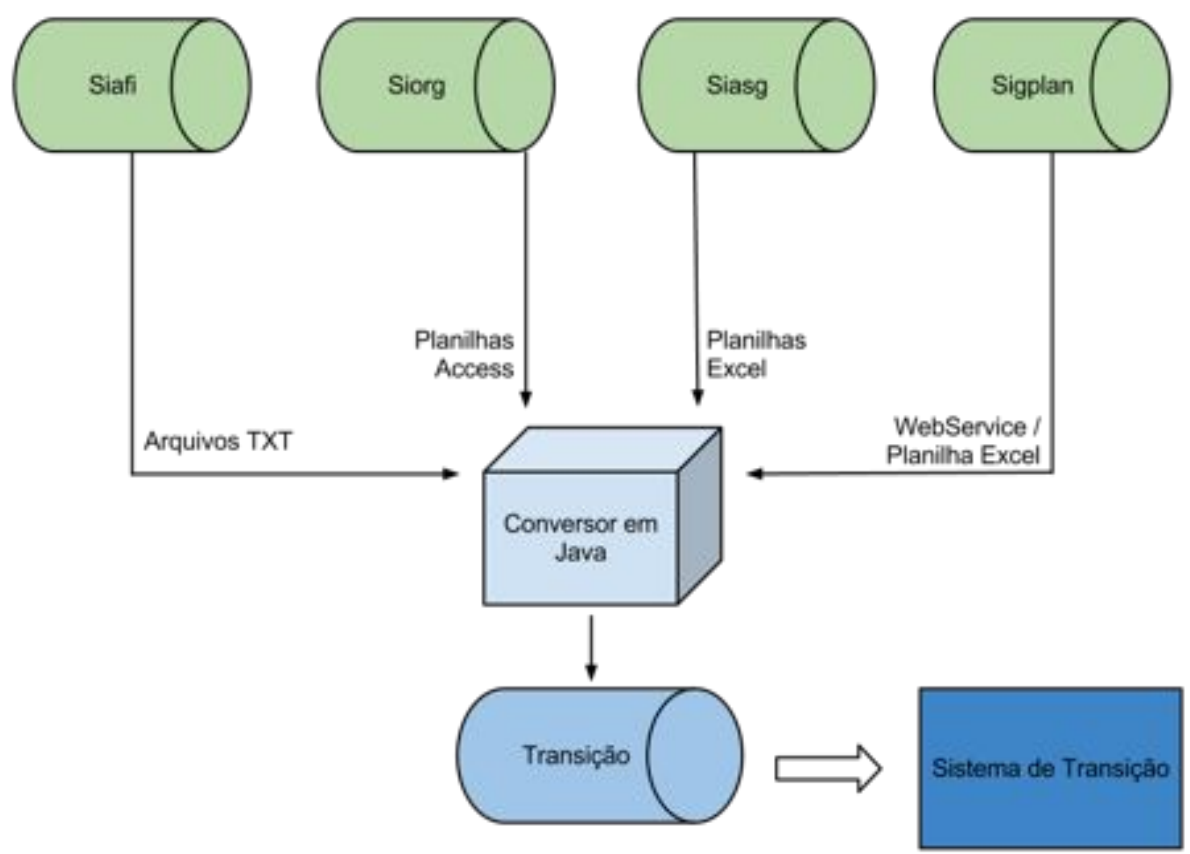

Figura 2. Integrações dos sistemas do governo e o Sistema de Transição 
Os sistemas que servem de entrada para o Sistema de Transição possuem formatos de armazenamento de dados diversos. Para facilitar o processo de importação, os formatos utilizados para a transmissão de dados foram convencionados. O Sistema SIAFI foi desenvolvido pela Secretaria do Tesouro Nacional (STN) em conjunto com o Serviço Federal de Processamento de Dados (SERPRO) visando integrar os sistemas de programação financeira, de execução orçamentária e de controle interno do Poder Executivo para fornecer informações gerenciais, confiáveis e precisas para todos os níveis da administração. Para a extração de dados do SIAFI, foi utilizado um extrator desenvolvido e disponibilizado pelo SERPRO. As consultas são realizadas pelo envio de todas as informações necessárias para a obtenção dos registros desejados. Após o término da extração, a saída é fornecida no formato de um arquivo de texto puro, que pode então ser utilizado para dar carga ao banco de dados do Sistema de Transição.

O sistema SIGPLAN consiste em um sistema estruturante que organiza e integra a rede de gerenciamento do PPA das diversas unidades orçamentárias do Governo Federal. De acordo com o Manual de Operação do SIGPLAN, esse sistema foi concebido para apoiar a execução dos programas, refletindo as características do modelo de gerenciamento: a orientação por resultados, a desburocratização, o uso compartilhado de informações, o enfoque prospectivo e a transparência para sociedade. Para a extração de dados do SIGPLAN, a equipe responsável disponibilizou inicialmente um serviço que continha as informações requeridas pelo Sistema de Transição. Entretanto, verificou-se que alguns dados fornecidos estavam inconsistentes com a sua fonte primária, ao longo do desenvolvimento do sistema. Dessa forma, optou-se por alterar o modo de transmissão dos dados para extrações em formato de planilhas Excel.

O Sistema Integrado de Administração de Serviços Gerais (SIASG) representa um conjunto informatizado de ferramentas para operacionalizar internamente $o$ funcionamento sistêmico das atividades inerentes ao Sistema de Serviços Gerais (SISG): gestão de materiais, edificações públicas, veículos oficiais, comunicações administrativas, licitações e contratos, do qual o MPOG é órgão central normativo. Para a extração dos dados deste sistema, contou-se com o apoio da equipe do Departamento de Logística e Serviços Gerais (DLSG) da Secretaria de Logística e Tecnologia da Informação (SLTI) do MPOG. Esta equipe realizava as extrações diretamente do Data Warehouse do sistema, na forma de planilhas Excel, que eram então repassadas para a equipe do Sistema de Transição.

O sistema SIORG tem como objetivos modernizar os sistemas de informações organizacionais da APF, proporcionar maior transparência da estrutura organizacional da APF para a sociedade e agilizar e tornar mais confiáveis as informações. Dessa forma, proporciona-se ao administrador melhor acompanhamento das políticas e diretrizes definidas pelo Governo Federal, contribuindo para o processo de Reforma do Estado e para recuperar em tempo real o histórico das estruturas organizacionais do Estado. Para realizar a importação dos dados necessários, a equipe do SERPRO disponibilizou as extrações necessárias através de tabelas Access.

Como pode ser identificado na descrição dos sistemas acima, os formatos dos dados que deviam ser integrados no Sistema de Transição eram diversos. Para auxiliar esse processo de importação, implementou-se um módulo em Java que continha a lógica da tradução dos dados de cada uma das diversas fontes para o formato utilizado pelo 
Sistema de Transição. O módulo possui como responsabilidade não apenas a leitura, limpeza, tradução e salvamento dos dados, mas também a capacidade de organizá-los e integrá-los de forma que o sistema Web desenvolvido possa executar as consultas de maneira otimizada. Por exemplo, os dados de orçamento dos órgãos são exibidos no Sistema de Transição apenas de forma sumarizada, porém são disponibilizados na fonte de forma bem mais detalhada. Assim, fica a cargo do módulo de conversão Java agrupar estes dados e armazená-los a fim de priorizar o desempenho da aplicação.

\section{Exemplo de Uso}

Nesta seção, é apresentado o Sistema de Transição por meio de exemplos referentes ao seu uso no ano de 2010. Cerca de 500 usuários cadastrados distribuídos em todos os órgãos APF foram responsáveis por validar e prover informações para compor o Livro de Transição. Em outubro de 2010, o sistema foi internalizado na Casa Civil, onde ficou disponível apenas para a Equipe de Transição e para geração do livro impresso. A seguir, algumas telas do sistema podem ser visualizadas. A Figura 3 exibe a tela inicial do sistema, apresentada após a autenticação do usuário, onde é possível escolher de qual órgão as informações devem ser exibidas e/ou alteradas.

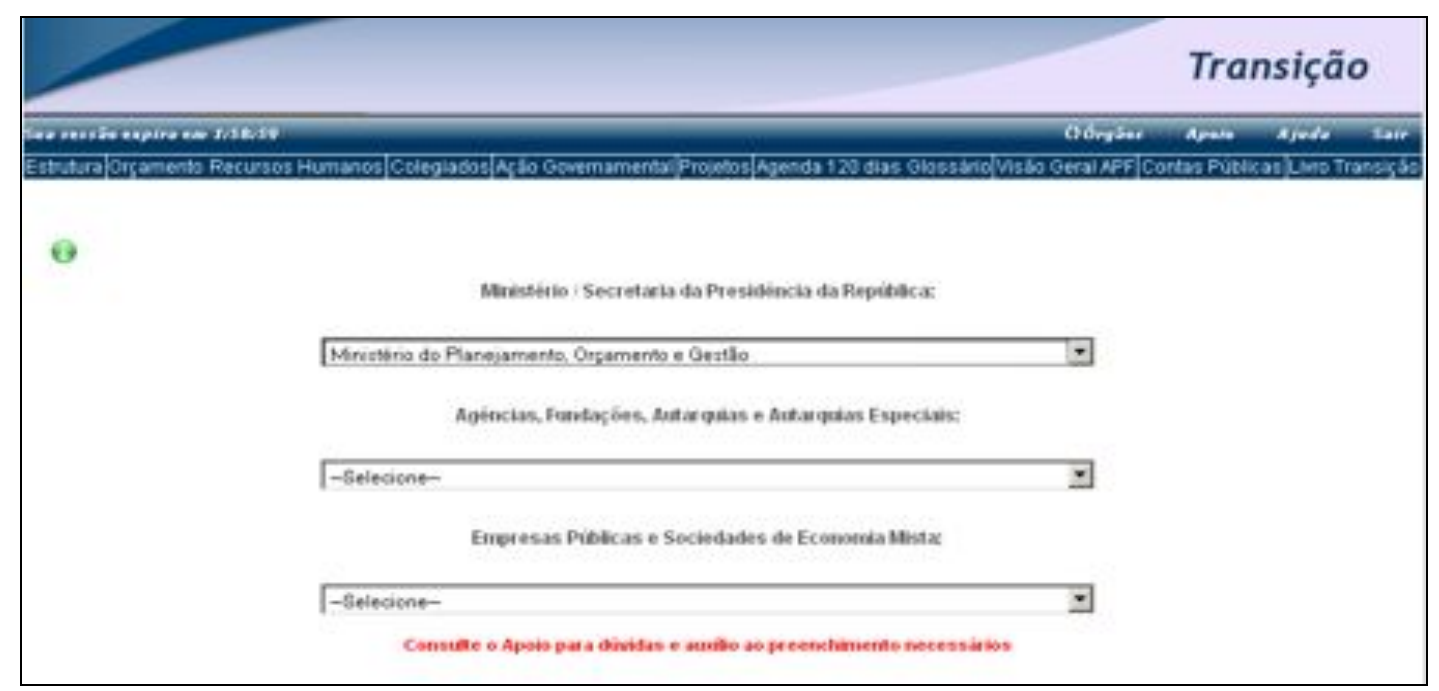

Figura 3. Interface de Seleção de Órgãos (Administração Direta e Indireta)

Na Figura 4, visualiza-se a tela de informações de Síntese Organizacional. Todos os dados são de somente leitura, uma vez que são oriundos dos sistemas externos e que não podem ser alterados no Sistema de Transição. Caso seja constatada qualquer necessidade de alteração de algum dado por parte do usuário, deve-se realizar no sistema de origem. Os dados, então, serão atualizados no Sistema de Transição na ocasião da carga de dados subsequente.

$\mathrm{Na}$ Figura 5, mostra-se a tela de informações de concursos públicos. São dispostos alguns dados oriundos de sistemas externos (que não podem ser alterados) e dados que são armazenados no Sistema de Transição. Esta tela representa a possibilidade de inserção de dados complementares aos dados existentes - grande parte, informações que são relevantes para a geração do Livro da Transição, mas inexistentes nos sistemas originais. 


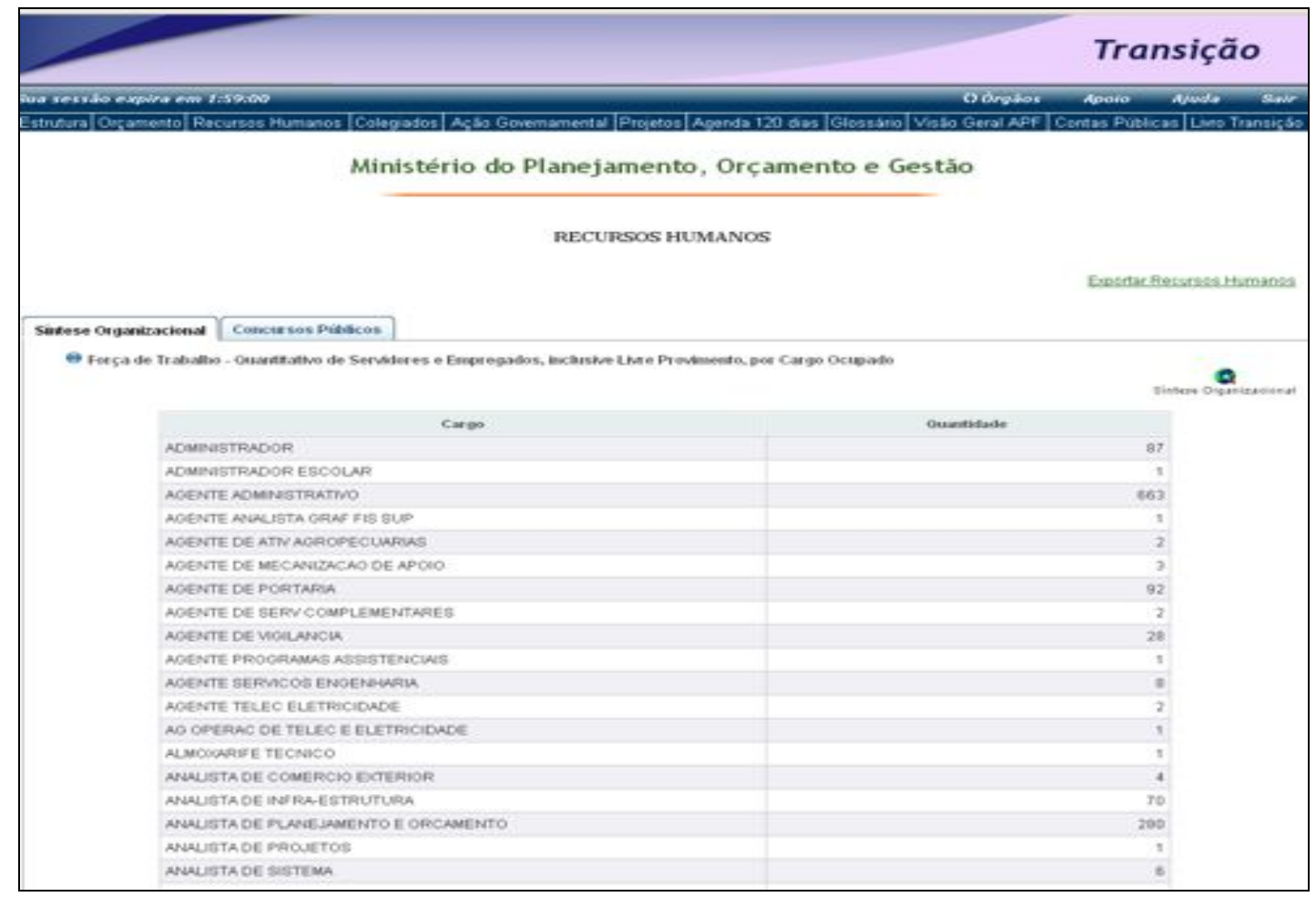

Figura 4. Interface de visualização das informações de Síntese Organizacional

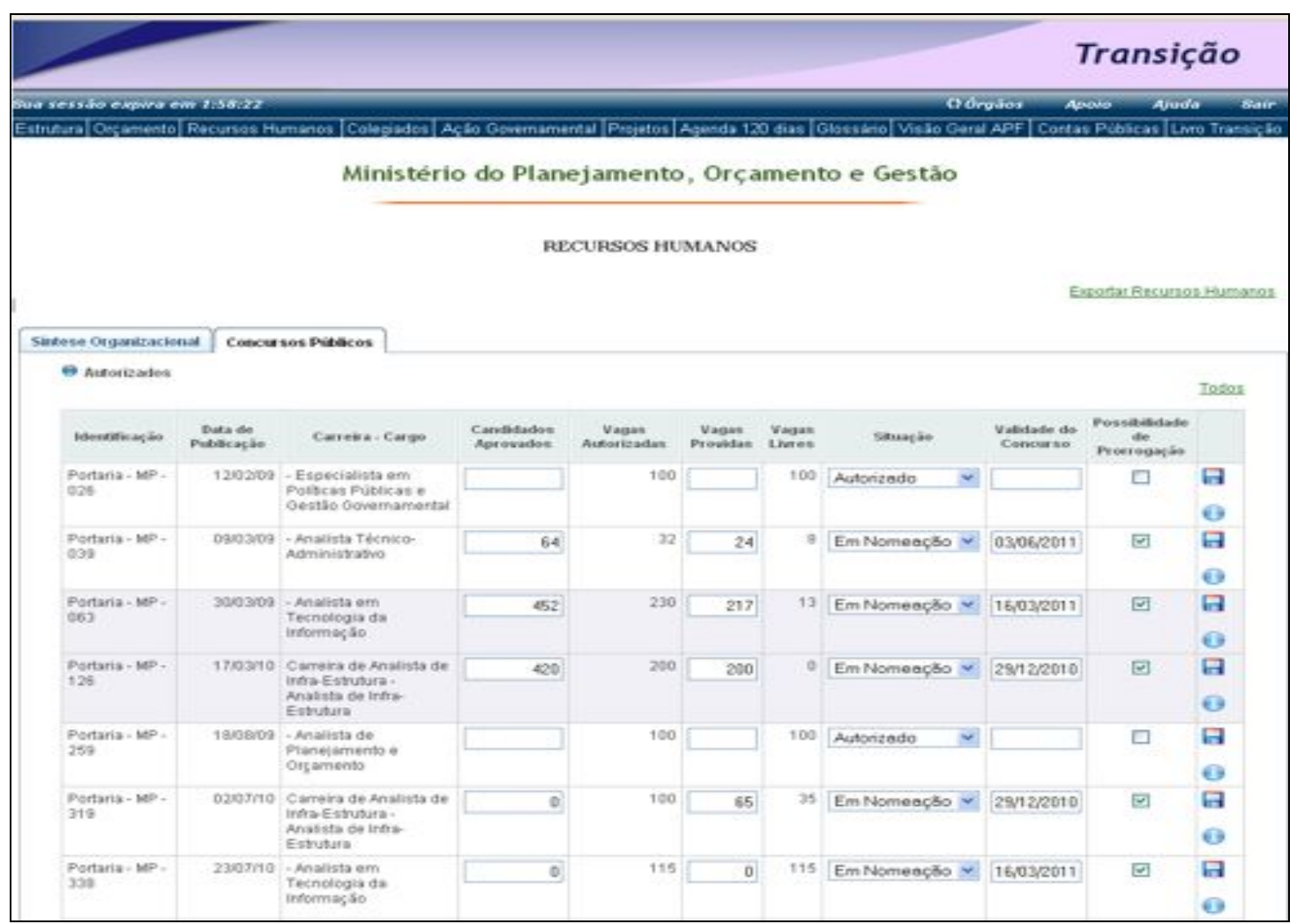

Figura 5. Interface de atualização/visualização das informações de concursos 


\section{Conclusão}

O crescimento da pesquisa em e-gov e a organização de sistemas em torno de ecossistemas digitais vem destacando a necessidade de manipulação de dados por meio da integração de diferentes sistemas. Frente ao uso de arquiteturas de referência e de serviços Web, este artigo apresentou o resultado de um projeto de pesquisa e desenvolvimento, que gerou o Sistema de Transição governamental. A implantação do sistema se deu com sucesso no fim de 2010, possibilitando o seu uso durante a transição de governos no início de 2011. Uma vez que existiu a constante preocupação com a validação do sistema com usuários chave de diferentes áreas do governo (i.e., usuários selecionados entre aqueles que usam o sistema, cujos cargos são variados), não foram relatados casos de resistência à sua implantação. No entanto, alguns setores não tiveram as suas necessidades atendidas plenamente pelo sistema e, assim, alguns ajustes posteriores no Livro da Transição precisaram ser realizados. Por exemplo, o orçamento da Casa Civil está reunido ao orçamento da Presidência e, portanto, deveria ser exibido apenas quando o órgão selecionado fosse Presidência, i.e., quando se seleciona Casa Civil, exibe-se um aviso para consultar o orçamento da Presidência. Essas alterações foram mapeadas e documentadas junto às alterações no Sistema de Transição para que, em uma versão futura, elas possam ser atendidas.

Um grande desafio no desenvolvimento do sistema consistiu na heterogeneidade dos sistemas a serem integrados. A falta de documentação, a rotatividade nas equipes envolvidas e o grande volume de dados que precisavam ser trocados representaram grandes riscos que tiveram de ser monitorados e mitigados durante todo o projeto. Um modelo mais robusto para a realização das cargas de dados foi proposto, utilizando a versão open source da ferramenta Talend, que possibilitou que a integração entre diferentes base de dados fosse feita através de um ambiente gráfico, facilitando a visualização do processo e a posterior manutenção. Este modelo já foi implementado para algumas integrações e, caso novas cargas sejam necessárias, as demais serão feitas sob demanda. Como trabalho futuro, pretende-se estabelecer um processo para que o sistema seja mantido e evoluído ao longo de um mandato, a fim de favorecer a eficácia na geração do Livro da Transição. Além disso, uma padronização da interface para importações de dados está sendo estudada a fim de fortalecer a autonomia do sistema.

\section{Referências}

Diniz, A., Silva, A. R., Sales, E., Reis, C, Reis, R. (2009) "SIGFAPESPA - Sistema Integrado de Gestão da Fundação de Amparo à Pesquisa do Estado do Pará", In: Anais do I Workshop de Computação Aplicada em Governo Eletrônico (WCGE), Bento Gonçalves, Brasil, pp. 1503-1515.

Franzosi, E. M., Garcia, A., Rodrigues, S. A., Blaschek, J. R., Souza, J. M. (2009) “Uma Proposta de Arquitetura Referencial SOA para Desenvolvimento de Sistemas para o Governo", In: Anais do I WCGE, Bento Gonçalves, Brasil, pp. 1417-1430.

Governo do Brasil (2012) "Extrator de Dados do Sistema Integrado de Administração Financeira do Governo Federal". Disponível em: <http://www.tesouro.fazenda. gov.br/siafi/imp_exp_dados.asp $>$. Acesso em jan/2013.

Jeong, C. (2007) "Fundamental of Development Administration”. Scholar Press. 
Martins, A. (2010) "Fundamentos de Computação Nuvem para Governos", In: Anais do Anais do II WCGE, Belo Horizonte, Brasil, pp. 716-728.

MPOG (2012) "Estratégia geral de Tecnologia da Informação - 2013-2015”, Ministério do Planejamento, Orçamento e Gestão, versão 1.1.

Nascimento, F. A. M., Lehnen, F. L., Moré, M. V., Leizer, S. A. (2009) “GIF: Sistema de Gestão e Inteligência Fiscal Orientado para a Administração Tributária Municipal”, In: Anais do I WCGE, Bento Gonçalves, Brasil, pp. 1489-1502.

Pita, M., Paixão, G. (2010) “Arquitetura de Busca Semântica para Governo Eletrônico", In: Anais do II WCGE, Belo Horizonte, Brasil, pp. 648-661.

Preti, J. P. D., Nunes, E. P. S., Filgueiras, L. V. L. (2010) “Arquitetura Crossmedia para Integração de Serviços de Governo Eletrônico", In: Anais do II WCGE, Belo Horizonte, Brasil, pp. 690-701.

Santanna, R. (2007) "Notícias - 10093 - Entrevista com Rogério Santanna”. Disponível em: <http://www.softwarelivre.org/news/10093>. Acesso em mai/2009.

Santos, R. P., Werner, C. M. L. (2011) "Gerência e Desenvolvimento de Sistemas de Informação na Realidade dos Ecossistemas de Software”, In: Anais do VII Simpósio Brasileiro de Sistemas de Informação, Minicursos, Salvador, Brasil, pp. 20-25.

Santos, R. P., Werner, C. M. L., Barbosa, O. A. L. P., Alves, C. F. (2012) "Software Ecosystems: Trends and Impacts on Software Engineering", In: Proceedings of the XXVI Brazilian Symposium on Software Engineering - Special Track 'Grand Challenges in Software/System Engineering', Natal, Brasil, pp. 206-210.

SIAFI (2012) “O que é o SIAFI”. Disponível em: <http://www.tesouro.fazenda.gov.br/ siafi/index_conheca_siafi.asp $>$. Acesso em jan/2013.

Silva, C. V. S., Ralha, C. G. (2010) "Utilização de Técnicas de Mineração de Dados como Auxílio na Detecção de Cartéis em Licitações”, In: Anais do II WCGE, Belo Horizonte, Brasil, pp. 595-608.

Sizo, A. M., Xisto, A. N. T., Fernandes, J. A. S., Santos, L. R. D., Martins, C. R. L. (2009) "Arquitetura PRODEPA: Uma solução para criação de sistemas distribuídos", In: Anais do I WCGE, Bento Gonçalves, Brasil, pp. 1431-1442.

Stefanuto, G., Alves, A. M., Spiess, M., Castro, P. F. D. (2011) "Quality in Software Digital Ecosystems: The User Perceptions", In: Proceedings of the 3nd ACM/IFIP International Conference on Management of Emergent Digital EcoSystems, San Francisco, USA, pp. 85-88.

TI MAIOR (2012) “Ecossistemas Digitais". Disponível em: < http://timaior.mcti.gov.br/ interna6.php $>$. Acesso em jan/13.

Tiboni, A. C., Lisboa, F. G. S., Mota, L. C. (2009) "Uma plataforma livre para padronização do desenvolvimento de sistemas no Governo Federal”, In: Anais do I WCGE, Bento Gonçalves, Brasil, pp. 1443-1449.

WCGE (2012) "Painel - Colóquio sobre um Programa de Pesquisa em Governo Eletrônico", In: Anais do IV WCGE, São Paulo, Brasil. 